\title{
Research Progress of Urban Public Service at Home and Abroad
}

\author{
Lanyan Wei \\ School of Civil Engineering and Architecture, Southwest University of Science and Technology, Mianyang 621010, Sichuan, China \\ Email: 1491694287@qq.com
}

\begin{abstract}
Public service facilities are an important part of China's harmonious and livable city construction and people's desire for a better life. The research topics of urban public service facilities allocation at home and abroad are different. It is found that foreign studies mainly focus on the location selection, accessibility, spatial equity and socio-economic effects of public service facilities allocation, while domestic studies pay more attention to the allocation standards of public service facilities and the optimization of layout, accessibility and spatial pattern. According to the characteristics of research at home and abroad, this paper reviews the literature respectively, and finally reflects on the differences of configuration research at home and abroad.
\end{abstract}

Keywords: configuration of public service facilities, spatial pattern, research progress

\section{Introduction}

Public service facilities refer to the space carriers that can provide various public goods and services for residents' daily life. They are public goods and services provided by urban public departments to the urban public, including investment and maintenance of urban infrastructure, provision and strengthening of jobs, social security services, and establishment and support of public utilities such as education, science and technology, culture, medical and health, sports, etc, Timely release social information to provide strong guarantee and create relevant conditions for the improvement of the quality of public life and participation in public affairs.

With the implementation of national development strategies such as equalization of public services, new urbanization and the construction of harmonious and livable cities, the allocation of urban public service facilities has attracted extensive attention. In view of this, this paper systematically combs the research progress and shortcomings of urban public service facility allocation at home and abroad, and puts forward some directions to be further studied in China's urban public service facility allocation, in order to provide scientific reference for further deepening the theory and method research of urban public service facility allocation in China.

\section{Research progress abroad}

Foreign research on the allocation of urban public service facilities mainly focuses on the topics of location selection, accessibility, spatial equity and socio-economic effects of the allocation of public service facilities.

The research on the location selection of public service facilities was first used to solve the actual layout of urban public service facilities. In the 21 st century, with the rapid combination of geographic information technology and location allocation model, the focus of foreign research on location selection of public service facilities has changed significantly. From the early research on the theoretical model of location selection of public service facilities to the synchronous development of spatial optimization theory and practical application. The combination of GIS technology and multi criteria decision analysis (MCDA) has further driven the research and development of location selection of public service facilities, making the multi-objective decision-making of facility location a reality. For example, Dell'ovo ${ }^{[1]}$ combined spatial analysis and MCDA method to conduct a detailed analysis on the location of medical and health care facilities in Milan, Italy, from four dimensions: functional quality, location quality, environmental quality and economic content.

In terms of accessibility, most studies only analyze the spatial distance barriers to the accessibility of public service facilities, and pay less attention to the restrictive effects of non spatial factors such as income and race on the accessibility of public service facilities. Relevant studies show that the accessibility of public service facilities is closely related to the actual utilization behavior of residents' facilities. Generally, the utilization rate of residents in areas with high accessibility of public service facilities is also high ${ }^{[2]}$.

Under the influence of the concept of spatial justice, western scholars are more and more interested in the research on the allocation of public service facilities in the areas of social vulnerable groups. The research on the spatial equity of public 
service facilities has become one of the hot topics of foreign academic attention in recent years, mainly testing the correlation between the accessibility of public service facilities and the characteristics of residents' socio-economic attributes. Many studies have found that areas with low socio-economic status are more vulnerable to the spatial unfairness of public service facilities, that is, the accessibility of public service facilities in areas where vulnerable groups gather is low or the service quality is poor. The research on the spatial evolution characteristics of Yokohama urban parks in Japan reveals that the unfair supply of local urban parks has always existed. The new parks are mainly built in affluent neighborhood areas and further attract the immigration of affluent groups.

At present, the research on the spatial equity of foreign urban public service facilities mainly focuses on its social equity dimension. Due to the different research areas, spatial scales, types of public service facilities and accessibility measurement methods, the research on the spatial equity of foreign urban public service facilities has not formed a unified conclusion.

In terms of socio-economic effects of public service facilities, some studies use the hedonic price model to analyze the capitalization effect of urban public service facilities allocation on house price or land price, and reveal the marginal utility value of different types of public service facilities.

\section{Domestic research progress}

The research on the allocation of urban community public service facilities in China mostly focuses on the problems of the facilities themselves, such as facility type, construction standard, facility location layout, facility allocation status evaluation, etc. more quantitative analysis methods are used in the research on facility location and satisfaction evaluation, and the research in other aspects more reflects the problems found Analyze the problems and put forward the research ideas of planning strategy.

The evaluation of facility location and layout mainly includes qualitative research and quantitative research. Qualitative research mainly summarizes the main problems existing in the existing layout of facilities through the investigation of the current situation. The problems mainly focus on the scattered distribution, uneven layout, most public welfare facilities are located in a partial position, insufficient public service facilities in areas far away from the central area, inconvenient use of the elderly, etc. ${ }^{[3-5]}$ Quantitative research mainly uses the methods of mathematical statistics and spatial analysis technology represented by GIS to analyze and evaluate the spatial distribution characteristics and social spatial correlation of facilities. For example, Junbo Gao and Chunshan Zhou ${ }^{[6]}$ used SPSS cluster analysis to realize the socio ecological analysis of the spatial allocation of urban community resources based on the special survey of urban public service facilities in Guangzhou and the traffic travel data and survey data of residents in the central urban area.

GIS software combined with algorithm analysis is widely used in the research of location selection to achieve more accurate location. Chunyan Zhao et al. ${ }^{[7]}$ realized the location of supermarkets, ATM machines and business centers by using GIS software + genetic algorithm. Shanshan $\mathrm{Yu}^{\left[{ }^{[8]}\right.}$ evaluated the accessibility of hospital space by using GIS software and two-step mobile search method, and optimized the spatial location of the hospital by using multi criteria decision analysis model.

The spatial distribution of public service facilities has a profound impact on the quality of life of local urban residents. Domestic scholars have discussed the spatial pattern of urban public service facilities in China from different spatial scales. Some studies show that, similar to China's socio-economic development level, the spatial pattern of urban basic public services in China also shows the characteristics of decreasing from the eastern coast to the central and western regions ${ }^{[9]}$. Other studies focused on the spatial pattern of urban public service quality in different provinces ${ }^{[10-11]}$. Some studies have also comprehensively analyzed the overall spatial distribution characteristics of different types of public service facilities ${ }^{[12]}$.

\section{Comments and prospects}

China is still in the primary stage of community construction. With the development of society and economy and the upgrading of community construction, the research needs to strengthen the research on the spatial law, social equity, performance evaluation and spatial optimization of public service facilities, and transfer the public service research to the relationship between users and facility entities. Foreign countries have earlier research and rich experience in this field. While learning from and referring to foreign advanced methods and achievements, China needs to strengthen the grasp of community characteristics and the characteristics of facility users, so as to form research methods and achievements with Chinese characteristics. In addition, it is necessary to increase the transformation from theoretical results to practical application in order to give full play to the practical value of research results. 


\section{References}

[1] Dell'Ovo M, Capolongo S, Oppio A. Combining spatial analysis with MCDA for the siting of healthcare facilities [J]. Land Use Policy, 2018; 76: 634-644.

[2] Park S J. Measuring public library accessibility:A case study using GIS [J]. Library \& Information Science Research, 2012; 34(1): 13-21.

[3] Zhitao Gong. Discussion on the allocation of public service facilities in residential areas of Tianjin [J]. Engineering construction and design, 2012; (S1): 93-95.

[4] Jianjie Shi, Xiaofang Zhu, Qiang Ma. Study on spatial layout planning of community public service facilities — Taking Runzhou District of Zhenjiang City as an example [C]. Planning Innovation: Proceedings of 2010 China urban planning annual conference. China Urban Planning Society, 2010: 2112-2120.

[5] Na Zhao, Runmin Liu, Hu Sai. Research on the construction of public service facilities in residential areas — Taking Ulanqab city as an example [J]. Inner Mongolia Science and technology and economy, 2014; (05): 13-14.

[6] Junbo Gao, Chunshan Zhou. Research on social differentiation of urban community resource allocation in transition period - An Empirical Study Based on Guangzhou [J]. Modern urban research, 2011; 26(07): 14-20.

[7] Chunyan Zhao, Jiping Li, Guohua Wang. Research on commercial location selection based on genetic algorithm [J]. Surveying and mapping information and engineering, 2010; 35(02): 8-9. Doi: 10.14188/j.2095-6045.2010.02.020.

[8] Shanshan Yu, Peng Peng, Xiaoqin Tian, Jieqiong Ding. Research on spatial layout and optimization of hospitals in Changsha Based on GIS [J]. Journal of Changsha University, 2012; 26(02): 90-94.

[9] Zenglin Han, Peng Du, Li Wang, Yang Yu, Dongxia Zhao, Yingxue Cong, Qilong Ren. Study on the optimal allocation method of regional public service facilities - Taking the allocation of Xinghua Street Primary School in Ganjingzi District, Dalian as an example [J]. Geographical Sciences, 2014; 34(07): 803-809. Doi: 10.13249/j.cnki.sgs.2014.07.010.

[10] Bin Li, Zenglin Han, Huiqiang Ma. Temporal and spatial analysis of the difference in the quality of urban basic public services in Liaoning Province [J]. Human geography, 2015; 30(03): 111-117. Doi: 10.13959/j.issn.10032398.2015.03.017.

[11] Weidong Shi, Lin Zhao. Measurement and spatial pattern characteristics of basic public service quality in Shandong Province [J]. Economic geography, 2015; 35(06): 32-37. Doi: 10.15957/j.cnki.jjdl.2015.06.005.

[12] Junbo Gao, Chunshan Zhou. Research on social differentiation of urban community resource allocation in transition period - An Empirical Study Based on Guangzhou [J]. Modern urban research, 2011; 26(07): 14-20. 